\title{
Spontaneous Hemorrhage from Renal Angiomyolipoma Pre- senting with Fever of Unknown Origin
}

\author{
Toshiro Sugimoto, Yoshikata Morita and Atsunori Kashiwagi
}

Key words: angiomyolipoma, fever of unknown origin, renal hemorrhage, tuberous sclerosis

\section{(DOI: 10.2169/internalmedicine.46.6361)}
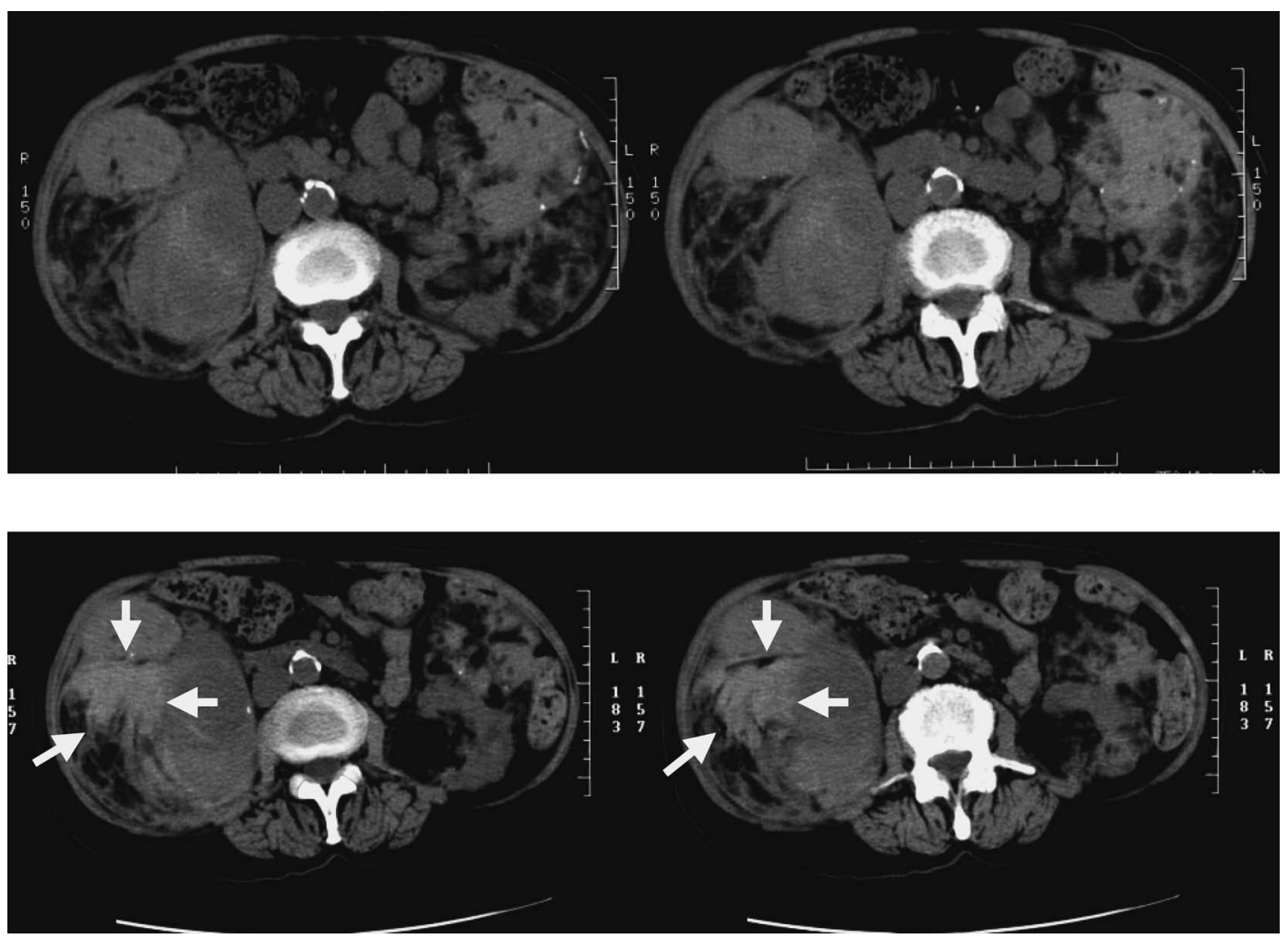

Picture 1. Abdominal computed tomography shows a large tumor containing fat, i.e., angiomyolipoma. High-attenuating material (arrows) suggestive of hematomas grows in the right renal tumor and perirenal space during hospitalization. (upper panel, day 0; lower panel, day 18).

A 74-year-old Japanese woman was admitted with a onemonth history of persistent fever. At the age of 54, the diagnosis of tuberous sclerosis was established because of facial angiofibromas and bilateral renal angiomyolipoma. She showed mild anemia and marked inflammatory signs, but evaluation for the presence of infection, including blood and urine cultures, yielded negative results. During hospitalization, her fever gradually disappeared with improvement of inflammatory signs. On the 18th hospital day, however, she had high fever with right flank pain and her anemia worsened. Abdominal computed tomography revealed features of recent growing hematomas in the right renal mass (Fig. 1). She rejected radiological intervention, but her symptoms were gradually improved without any treatments, and she was discharged. Although it has been reported that angiomyolipoma per se can cause fever in tuberous sclerosis patients 
by an unknown mechanism, we concluded that her fever might have been caused by spontaneous hemorrhage from renal angiomyolipoma because hematomas as a source of fever have been well recognized. Hemorrhage typically occurs in patients with a large angiomyolipoma (4 $\mathrm{cm}$ or greater) presenting with life-threatening conditions (e.g., hemorrhage shock); however, the present case indicates that hemorrhage from renal angiomyolipoma can emerge as fever of unknown origin.

(C) 2007 The Japanese Society of Internal Medicine http://www.naika.or.jp/imindex.html 